\title{
Developing novel drugs for systemic lupus erythematosus. Lessons learned from the belimumab trials
}

Cristina Pamfil 1,2, Antonis Fanouriakis ${ }^{2}$, Dimitrios T. Boumpas 2,3

1 Department of Rheumatology, "Iuliu Hatieganu" University of Medicine and Pharmacy Clui, Romania; EULAR Scholar Department of Rheumatology, Clinical Immunology and Allergy, University Hospital of Heraklion, Greece

2 Department of Rheumatology, Clinical Immunology, and Allergy, University Hospital of Heraklion, Greece

3 Institute of Molecular Biology and Biotechnology, FORTH, Heraklion, Greece

\section{Abstract}

Systemic lupus erythematosus is the prototypic autoimmune disease with a broad range of clinical manifestations and a complex pathogenesis. B-cells hold a central role in its pathogenesis, not only as autoantibody producing cells, but also by producing other inflammatory mediators and by presenting autoantigens to autoreactive T cells. BlyS, a soluble ligand of the TNF cytokine family, is a key factor affecting B-cell homeostasis and survival and its blockade ameliorated the disease in animal models and preclinical studies of SLE. Following an unsuccessful phase II trial of belimumab, a monoclonal antibody targeting BlyS, two large phase III studies in patients with mild-to-moderate disease, BLISS-52 and BLISS-76, met their primary endpoints showing better efficacy of the drug over standard of care alone. To this end, development of a novel more sensitive responder index and improvements in study designs were crucial. As a result, belimumab became the first drug to get approval for the treatment of SLE after more than 50 years. In this paper we discuss the rationale, development, indications, lessons learned, pitfalls and challenges for this novel therapy and pointout to additional issues that need to be addressed in the future.

\section{Keywords}

Belimumab; Systemic lupus erythematosus; Randomized controlled trials

Corresponding author

Dr. Dimitrios T. Boumpas, MD, FACP

Departments of Internal Medicine and Rheumatology,

Clinical Immunology and Allergy,

University of Crete Medical School, Voutes, Heraklion,

PO Box 2209, Greece

Phone: (+30) 2810394635

boumpasd@med.uoc.gr
Disclosure

The Authors declare that they have no financial competing interest related to the contents of this article 


\section{Introduction}

Systemic lupus erythematosus (SLE) represents the prototypic systemic autoimmune disease. Although not as common as rheumatoid arthritis (affects approximately 30-50 subjects per 100,000), nevertheless it causes wide spread inflammation involving almost every single organ including the joints, the skin, the kidneys, the blood, the brain, the lungs or the heart. Lupus is associated with substantial annual direct cost burden at par with other major rheumatologic diseases such as rheumatoid arthritis in terms of health care resource utilization as well as losses of productivity due to work capacity impairment $[1,2]$.

Although the care of patients with SLE has improved over the years, current state-of-the-art therapy is far from optimum. Most available therapeutic agents mainly aim at dampening signs and symptoms of the disease and are often accompanied by considerable toxicity, let alone the fact that most of them are not FDA-approved for treatment of the disease. Failure to respond, partial response, flares or significant toxicities still plague modern lupus therapeutics.

SLE has turned out to be a challenging disease for the development of new therapeutic agents. Demonstration of efficacy of candidate therapies has been hampered by heterogeneity in disease phenotype, concomitant immunosuppressive medication and lack of unequivocal hard endpoints for lupus trials. Rituximab (monoclonal antibody targeting CD20 on B-cells) represents an illustrative example. While extensive evidence from observational studies has suggested that the drug works in the clinical practice, two randomized controlled trials (RCTs) in renal and extrarenal lupus failed to reach their primary endpoints of superior efficacy over standard of care [3,4]. Another B-cell inhibitor, atacicept, did not fair as well too. Atacicept is a soluble, fully human, recombinant fusion protein that inhibits B-cell stimulating factors APRIL (a proliferation-inducing ligand) and BLyS (B-lymphocyte stimulator, also known as B-cell activating factor belonging to the TNF-family, BAFF). In the APRIL-LN study, aimed at evaluating the efficacy and safety of atacicept in patients with active lupus nephritis (LN), the trial was terminated after the enrolment of six patients, due to an unexpected decline in serum immunoglobulin $\mathrm{G}(\mathrm{IgG})$ and the occurrence of serious infections [5]. In this context, the recent success of belimumab, a fully human monoclonal antibody targeting BlyS, has rekindled interest in targeting B-cells to treat the disease.

In this paper, we review the rationale and clinical development of the drug.

\section{BLyS biology and its role in murine and human lupus}

BLyS is a soluble ligand of the TNF superfamily and, together with APRIL, plays a role in the selection and survival of B-cells. These ligands bind to three different receptors on the surface of B-cells but with different specificities, which accounts for their non-redundant biologic actions. BLyS interacts with all three receptors, namely BR3 (BAFF receptor 3, also known as BAFF receptor), TACI (transmembrane activator and calcium modulator and cyclophylin ligand interactor), and BCMA (B-cell maturation antigen) [6]. On the other hand, APRIL signals only through TACI and BCMA. Intracellular signaling after ligation of each of these receptors leads mainly to activation of the nuclear factor-kB (NF-kB) pathways (nonclassical for BR3/classical for TACI and BCMA), ultimately leading to B-cell survival through inhibition of apoptosis.

The selectivity of BLyS ligation to BR3 has important implications for its actions during B-cell development. BLyS-BR3 interactions are crucial for the survival of transitional B-cells, as these cells migrate from the bone marrow to the periphery. This level of differentiation represents a crucial checkpoint for the elimination of potentially autoreactive B-cells prior to their maturation in the lymph nodes [7]. The antiapoptotic effect of BLyS during this phase may facilitate the escape of such cells from mechanisms 


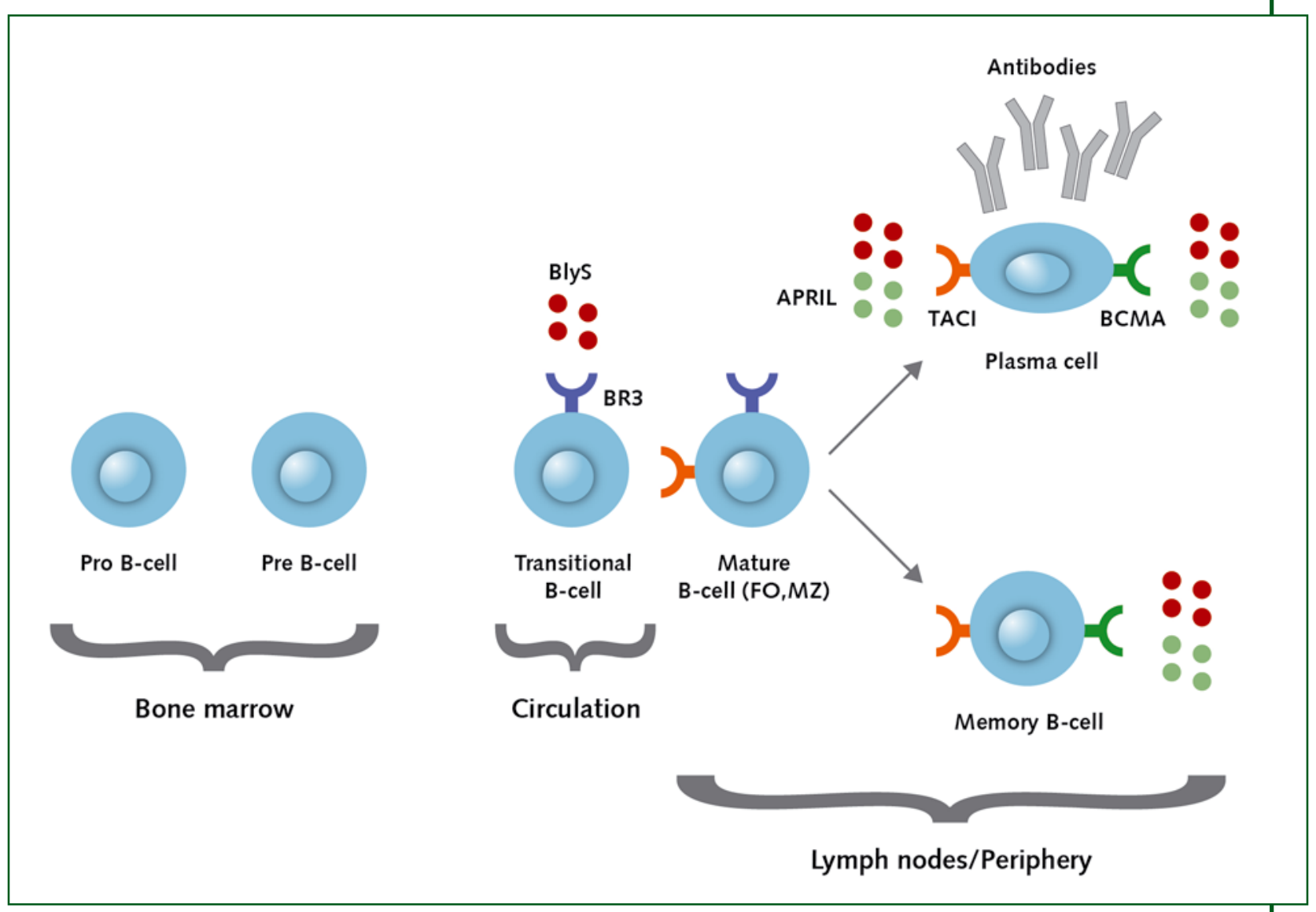

Figure 1. Differential receptor expression during subsequent phases of B-cell development. Before exiting the bone marrow, B-cells undergo a delicate selection process to prevent the escape of autoreactive cells through mechanisms such as clonal deletion and receptor editing. After this point, surviving cells become transitional B-cells that migrate to peripheral lymphoid organs to become follicular or marginal zone B-cells. Exposure to antigen will ultimately lead to the final stage of maturation with the development of memory B-cells and antibody-producing plasma cells. BLySBR3 interactions are crucial in the transitional stage, wherein they provide anti-apoptotic signals that may allow potentially autoreactive cells to escape deletion. At later stages of development, APRIL-TACI and APRIL-BCMA interactions become more important.

$\mathrm{BCMA}=\mathrm{B}$-cell maturation antigen; $\mathrm{FO}=$ follicular; $\mathrm{MZ}=$ marginal zone; $\mathrm{TACl}=$ transmembrane activator and calcium modulator and cyclophylin ligand interactor

of central tolerance. In subsequent developmental stages following exposure to antigens, B-cells seem to depend more on the presence of TACI and BCMA, rather than on the combined effect of BlyS and APRIL. Indeed, memory B-cells and antibody-producing plasma cells depend more on the presence of APRIL, not only for survival but also for isotype class-switching [8,9]. Consequently, BLyS inhibition is associated with depletion of naïve and transitional B-cells, while blockade of APRIL has a profound effect on plasma cells and immunoglobulin production.

In SLE, the role of B-cells extends beyond generation of pathogenic autoantibodies, which may either be directly cytotoxic or promote tissue injury through the formation and deposition of immune complexes. B-cells may regulate autoreactive T-cell function by means of competent antigen-presentation and costimulation, as well as mediate the production of inflammatory cytokines. Various abnormalities in B-cell subsets have been described in SLE, and active disease is accompanied by depletion of naïve B-cells and expansion of peripheral memory and plasma B-cells [10]. 
Studies in animal models have provided important insights regarding the role of B-cell survival factors, especially BLyS in lupus. Overexpression of BLyS in transgenic mice results in an lupus-like autoimmune phenotype with lymphoid hyperplasia, development of autoantibodies against nuclear antigens, and deposition of immune complexes to the kidney [11]. In two mouse models of lupus nephritis, blockade of BLyS (either selective or in combination with APRIL blockade) was able to prevent and treat the disease and deplete B-cells; the formation of autoantibodies was modestly affected $[12,13]$. Inhibition of BLyS preferentially targeted early stage B-cells, leaving memory and plasma cells relatively intact [14]. Following data from animal studies, early human studies showed that BlyS is overexpressed in patients with SLE and other autoimmune diseases [15]. More interestingly, BLyS concentrations are associated with changes in SLE disease activity and anti-dsDNA antibody titers [16,17]. These findings provided strong rationale for a phase I study of belimumab, a human $\operatorname{IgG}_{1}$ monoclonal antibody that binds soluble human BLyS, in SLE patients.

\section{The development of belimumab}

\section{The phase I trial}

The phase I double-blind RCT aimed at establishing the safety, pharmacokinetics and biological activity of belimumab [18]. The trial enrolled 70 SLE patients with mild-to-moderate disease activity treated with four different drug doses $(1,4,10$ and $20 \mathrm{mg} / \mathrm{kg})$, administered as single $(\mathrm{n}=34)$ or two infusions $(\mathrm{n}=36) 21$ days apart. Patients were followed over a 84-105-day period. Tolerability and safety were ascertained by no significant increase of infections or other adverse events compared to placebo. The pharmacokinetics were linear across the 1.0 to $20 \mathrm{mg} / \mathrm{kg}$ dose and the profile was consistent with a fully human antibody. The estimated half-life of 14 days of the drug suggested a 28-day dosing protocol

\begin{tabular}{|c|c|}
\hline $\begin{array}{l}\text { Disease } \\
\text { outcome measure }\end{array}$ & Comments \\
\hline SELENA-SLEDAI* & $\begin{array}{l}\text { Measure of disease activity } \\
\text { Assesses } 24 \text { disease manifestations, that are each atributed a score }(1-8) \text {. The score ranges from } \\
0-105 \text {, but scores }>20 \text { are uncommon } \\
\text { The cummulative score reflects the severity of disease activity } \\
\text { Commonly used in every day clinical practice }\end{array}$ \\
\hline BILAG & $\begin{array}{l}\text { Complex assessment tool of disease activity of individual organ domains } \\
\text { Assesses } 86 \text { disease manifestations of } 8 \text { organ domains } \\
\text { Each organ domain is given a score of A (severe disease), B (moderate disease), C (mild, stable } \\
\text { disease), D (no current disease activity in a previously affected organ system), or E (the organ system } \\
\text { has never been involved) } \\
\text { Used predominantly in clinical trials }\end{array}$ \\
\hline ECLAM & $\begin{array}{l}\text { Measure of activity of } 15 \text { weighted clinical and serological items } \\
\text { Disease activity scores range from } 0 \text { to } 10\end{array}$ \\
\hline PGA & Physician's subjective measure of the patient's general health status on a visual analogue scale $(0-3)$. \\
\hline SRI & $\begin{array}{l}\text { Composite outcome measure that comprises SELENA-SLEDAI scores, BILAG domain scores and PGA } \\
\text { Used as primary outcome measure in clinical trials }\end{array}$ \\
\hline
\end{tabular}

Table I. SLE outcome measures. Adapted from [19]

BILAG = British Isles Lupus Assessment Group; ECLAM = European Consensus Lupus Activity Measure;

PGA = Physician Global Assressment; SELENA-SLEDAI = Safety of Estrogen in Lupus National

Assessment-Systemic Lupus Erythematosus Disease activity Index; SRI = SLE Responder Index 
for future trials. The biologic activity of belimumab was reflected by the significant reduction of peripheral CD20 ${ }^{+}$B-cells in the combined group of patients treated with a single dose of belimumab versus placebo (day $42 \mathrm{p}=0.0042$; day $84 \mathrm{p}=0.0036$ ), as well as in patients treated with two doses of belimumab versus placebo (day 105; $\mathrm{p}=0.0305$ ). However, the trial did not meet the secondary endpoint of efficacy; there was no significant improvement in SELENA-SLEDAI (SS) scores, physician global assessment (PGA) or SELENA-SLEDAI Flare Index (SFI) in the belimumab treated population compared to placebo. The limited number of infusions, short duration of study and inclusion of patients with low or no disease activity were thought to account for the negative findings.

\section{The phase II trial}

The subsequent phase II RCT enrolled 449 SLE patients to assess safety, tolerability, biologic activity and efficacy of belimumab [19]. The patients were assigned to belimumab $(1,4$ or $10 \mathrm{mg} / \mathrm{kg})$ or placebo, administered every 28 days in a 52 -week study. Inclusion criteria were patients with active disease defined by a SS score of at least 4 and a history of measurable autoantibodies (but not required to be present at screening). A 60-day stable standard of care treatment consisting of prednisone, antimalarial and immunosuppressive agents was requested until the screening date, and was afterwards allowed to be adapted upon clinical need. Key exclusion criteria were active lupus nephritis or central nervous system involvement. The trial reached neither of its primary endpoints namely changes in the SS scores, flare rates and time to first flare. Thus, no differences in disease activity at week 24 or at week 52 between the belimumab and placebo groups were recorded and no dose-dependent effects were observed. No differences in flare rates or time to flare were found; however, an analysis of time to first flare starting at week 24 through week 52 revealed a more prolonged time to flare in the belimumab group versus placebo ( 154 versus 108 days; $p=0.0361$ ). This finding suggested that belimumab could stabilize disease activity, but had a latency period until clinically measurable efficacy is demonstrated.

At a post-hoc analysis of patients with serologically inactive disease $(\mathrm{n}=128)$ were compared to serologically active patients defined as ANA $>1: 80$ and/or anti-dsDNA $>30 \mathrm{IU} / \mathrm{ml}$ at the time of screening $(\mathrm{n}$ = 321) [20]. Serologically active patients had more major organ involvement (renal 19\% versus 34\%; hematologic 33\% versus 59\%), higher mean SS scores (8.9 versus 9.8) and more frequent prednisone use $(\mathrm{p}=0.002)$. The same patients had lower complement levels $(\mathrm{p}<0.0001)$, higher levels of immunoglobulins ( $\mathrm{p} \leq 0.001)$, as well as a lower baseline of $\mathrm{CD} 19^{+} / \mathrm{CD} 20^{+} \mathrm{B}$-cell counts $(\mathrm{p} \leq 0.01)$ and higher levels of BlyS ( $p<0.0001$ ). These features defined a patient subset with evidence of B-cell hyperactivity, more likely to respond to an anti-BlyS drug.

\section{SLE responder index (SRI)}

In response to the phase II trial, an evidence-based exploratory analysis was conducted to develop a composite disease activity index to be used as primary efficacy endpoint in lupus trials. The SLE responder index (SRI) comprises SS scores to establish global improvement, BILAG domain scores to reflect worsening of previous unaffected systems and PGA to ensure that improvements in disease activity are not achieved at the expense of the patient's overall condition. According to the SRI, response to therapy was defined as [21]:

- $\geq 4$-point reduction from baseline in SS score;

- no new BILAG A organ domain or

- $\geq 2$ new BILAG B organ domain scores compared with baseline, and $c)$ no worsening in PGA $(<0.3$ point increase from baseline).

With SRI set as the primary endpoint, a retrospective analysis of the phase II study revealed that in serologically active patients SRI response rates were significantly higher at week 52 in the belimumab- 
treated group versus placebo ( $46 \%$ versus $29 \%$; $\mathrm{p}=0.006)$. Moreover, an analysis of all patients $(\mathrm{n}=$ 449) irrespective of baseline autoantibody status, exhibited a significantly higher SRI response rate at week 52 in the belimumab treatment group versus placebo ( $45.9 \%$ versus $35.4 \%$; $p=0.045$ ), suggesting clinical efficacy of the drug.

\section{Phase III: study design and endpoints}

During phase III, belimumab was evaluated in two large double-blind, multicenter, RCTs: BLISS-52 ( $\mathrm{n}=$ 865) and BLISS-76 (N=819) [22,23]. The BLISS trials (a Study of Belimumab in Subjects with Systemic Lupus Erythematosus) were conducted concomitantly worldwide, covering different geographical areas: Latin America, Asia-Pacific and Eastern Europe in BLISS-52, and North America and Europe in BLISS-76.

Study design

The studies had identical study designs and endpoints which reflected the phase II trial experience. Patients on standard of care were randomly assigned to receive either placebo or $1 \mathrm{mg} / \mathrm{kg}$ or $10 \mathrm{mg} /$ $\mathrm{kg}$ belimumab by intravenous infusion on days $0,14,28$ and then every 28 days through week 48 in BLISS-52, and week 72 in BLISS-76 trial. Eligible for enrollment were active SLE patients with a SS score $\geq 6$ and seropositivity defined by 2 positive ANA or anti-dsDNA test results (ANA titer $\geq 1: 80$ and /or anti-dsDNA antibody level $\geq 30 \mathrm{IU} / \mathrm{ml}$ ) of which $\geq 1$ test had to be obtained during the screening. A stable treatment regimen for at least 30 days (excluding biological therapies or cyclophosphamide) before baseline was required. Changes in concurrent medication were restricted for immunosuppressants after week 16 and for antimalarials and prednisone after week 24. Moreover, the prednisone dose had to return to within $25 \%$ or $5 \mathrm{mg}$ greater than the baseline dose, with no further increases during the study. In case of adjustments in concurrent medication outside the defined parameters (such as increases in immunosuppressants that could reflect disease worsening), patients were declared treatment failures.

\section{Study endpoints}

The primary endpoint was the response rate at week 52, assessed by the SRI. Major secondary endpoints included: proportion of patients with $\geq 4$ point reduction from baseline in SS score at week 52, mean change in PGA score at week 24, mean change in SF-36 physical component summary score at week 24 and proportion of patients with an average reduction in prednisone dose $\geq 25 \%$ from baseline to $7.5 \mathrm{mg}$ /day or less during weeks $40-52$.

\section{General characteristics: BLISS-52 versus BLISS-76}

The overall baseline demographics of the two trials were comparable. The trial populations were similar with regard to organ involvement and disease activity. Table II compares the baseline characteristics of the two studies with emphasis on dissimilarities. There were differences in the background medication and serological activity that may account, at least in part, for the lower response rates recorded in BLISS-76 compared to BLISS-52.

\section{Efficacy}

Both BLISS-52 and BLISS-76 met their primary endpoints as assessed by the SRI. Clinical relevance was reached in a dose-dependent manner and was uneven throughout the trials. Treatment with belimumab $10 \mathrm{mg} / \mathrm{kg}$ influenced all three SRI components, while treatment with belimumab $1 \mathrm{mg} / \mathrm{kg}$ had an effect on two SRI components; clinical benefit was best reflected by a $\geq 4$ point reduction in the SS score. Higher response rates were obtained with belimumab $10 \mathrm{mg} / \mathrm{kg}$ compared to standard of care alone at 52 weeks in both trials (57.6\% versus 43.6\%; $\mathrm{p}=0.0006$ in BLISS-52, and 43.2\% versus 33.5\%; $\mathrm{p}=0.017$ in BLISS-76). When treated with $1 \mathrm{mg} / \mathrm{kg}$ belimumab and compared to placebo, more patients responded in BLISS-52 (51.5\% versus $43.6 \%, \mathrm{p}=0.013)$, but not in BLISS-76 (46.2\% versus 38.8\%, $\mathrm{p}=0.089$ ). However, pooled analysis of the two trials yielded significant differences at week 52 for both belimumab treatment arms 


\begin{tabular}{|c|c|c|c|c|c|c|}
\hline \multirow[b]{2}{*}{$\begin{array}{c}\text { Baseline } \\
\text { characteristics }\end{array}$} & \multicolumn{3}{|c|}{ BLISS-52 } & \multicolumn{3}{|c|}{ BLISS-76 } \\
\hline & $\begin{array}{l}\text { Placebo } \\
(n=287)\end{array}$ & $\begin{array}{c}\text { Belimumab } \\
1 \mathrm{mg} / \mathrm{kg} \\
(\mathrm{n}=288)\end{array}$ & $\begin{array}{c}\text { Belimumab } \\
10 \mathrm{mg} / \mathrm{kg} \\
(\mathrm{n}=290)\end{array}$ & $\begin{array}{l}\text { Placebo } \\
(n=275)\end{array}$ & $\begin{array}{c}\text { Belimumab } \\
1 \mathrm{mg} / \mathrm{kg} \\
(\mathrm{n}=271)\end{array}$ & $\begin{array}{c}\text { Belimumab } \\
10 \mathrm{mg} / \mathrm{kg} \\
(\mathrm{n}=273)\end{array}$ \\
\hline \multicolumn{7}{|l|}{ Race } \\
\hline Indigenous American & $89(31 \%)$ & $98(34 \%)$ & $92(32 \%)$ & $36(13 \%)$ & $33(12.2 \%)$ & $34(12.5 \%)$ \\
\hline White & $82(29 \%)$ & $76(26 \%)$ & $71(24 \%)$ & $188(68 \%)$ & $192(71 \%)$ & $189(69 \%)$ \\
\hline Black American & $11(4 \%)$ & $8(3 \%)$ & $11(4 \%)$ & $39(14.2)$ & $40(15 \%)$ & $39(14 \%)$ \\
\hline Asian & $105(37 \%)$ & $106(37 \%)$ & $116(40 \%)$ & $11(4 \%)$ & $6(2.2 \%)$ & $11(4 \%)$ \\
\hline Hispanic or Latino & $143(50 \%)$ & $141(49 \%)$ & $136(47 \%)$ & $55(20 \%)$ & $62(22.9 \%)$ & $56(20.5 \%)$ \\
\hline \multicolumn{7}{|l|}{ Biomarkers } \\
\hline Anti-dsDNA $\geq 30 I U / m l$ & $205(71 \%)$ & $221(77 \%)$ & $218(75 \%)$ & $174(63 \%)$ & $171(63 \%)$ & $179(66 \%)$ \\
\hline C3 below lower limit of normal & $132(46 \%)$ & $148(51 \%)$ & $147(51 \%)$ & $116(42 \%)$ & $100(37 \%)$ & $115(42 \%)$ \\
\hline C4 below lower limit of normal & $160(56 \%)$ & $173(60 \%)$ & $180(62 \%)$ & $143(52 \%)$ & $141(52 \%)$ & $147(54 \%)$ \\
\hline \multicolumn{7}{|l|}{ Medications } \\
\hline Daily prednisone use & $276(96 \%)$ & $276(96 \%)$ & $278(96 \%)$ & $212(77 \%)$ & $211(78 \%)$ & $200(73 . \%)$ \\
\hline$>7.5 \mathrm{mg} /$ day prednisone use & $192(67 \%)$ & $204(71 \%)$ & $204(71 \%)$ & $126(46 \%)$ & $130(48 \%)$ & $120(44 \%)$ \\
\hline Any immunosuppressant use & $122(43 \%)$ & $120(42 \%)$ & $123(42 \%)$ & $154(56 \%)$ & $153(56 \%)$ & $148(54 \%)$ \\
\hline
\end{tabular}

Table II. Baseline characteristics of patients in BLISS-52 and BLISS-76

(responder rate: $50.6 \% \mathrm{p}<0.0001$ with $10 \mathrm{mg} / \mathrm{kg}$ belimumab, $46.2 \% \mathrm{p}=0.006$ ) with $1 \mathrm{mg} / \mathrm{kg}$ belimumab versus $38.8 \%$ with placebo) $[24,25]$. Of note, the difference in the rate of SRI between treatment and placebo became apparent as soon as week 16, but it was not maintained through week 76 [23].

Flares

A secondary endpoint of the studies was flare rate and time to flare as measured with SLE Flare Index (SFI). Considerable improvements were seen in both belimumab groups of the BLISS-52, but the effect remained mild in BLISS-76 [26,27]. Pooled analysis of the two studies came to strengthen the findings: compared to placebo (23.7\%), belimumab treated patients developed less flares, including less severe flare rates $(17 \%$ in the $1 \mathrm{mg} / \mathrm{kg}$ belimumab group, $\mathrm{p}<0.05 ; 15.6 \%$ in the $10 \mathrm{mg}$ belimumab, $\mathrm{p}$ $<0.01)$ and a prolonged time to first flare in both treatment groups [24]. The effect was present in a dose-dependent manner. Of note, new BILAG $1 \mathrm{~A}$ or $2 \mathrm{~B}$ score rates were reduced only in the $10 \mathrm{mg} / \mathrm{kg}$ belimumab arm compared to placebo.

\section{Steroid sparing effect}

A secondary endpoint of the studies was the proportion of patients with an average reduction in prednisone dose $\geq 25 \%$ from baseline to $7.5 \mathrm{mg}$ /day or less during weeks 40-52. In BLISS-52, a steroid sparing effect was observed in $21 \%$ of patients on belimumab $1 \mathrm{mg} / \mathrm{kg}$ compared to $12 \%$ placebo ( $\mathrm{p}=$ 0.0252) [22,26]. In BLISS-76, although numerically more patients achieved steroid dose reduction in belimumab treatment groups, a statistically significant effect was not demonstrated. Of note, a reduction by $\geq 25 \%$ with belimumab $10 \mathrm{mg} / \mathrm{kg}$ or by $\geq 50 \%$ at week 52 was not reached [24]. Combined analysis of both trials established a steroid sparing effect in $20 \%$ of patients on $1 \mathrm{mg} / \mathrm{kg}$ belimumab (p $=0.0097)$ and $18 \%$ of patients on $10 \mathrm{mg} / \mathrm{kg}$ belimumab $(\mathrm{p}=0.0045)$ compared to $12 \%$ of patients on standard of care alone [24].

\section{Biomarkers}

The most robust effect of belimumab in SLE was demonstrated in the domain of activity biomarkers. Elevated IgG levels at baseline were markedly reduced in both belimumab treatment groups in 
BLISS-52 and BLISS-76. Combined analysis of the two trials showed that by week 52, $49.2 \%$ of patients in the belimumab $1 \mathrm{mg} / \mathrm{kg}$ and $10 \mathrm{mg} / \mathrm{kg}$ treatment arms achieved normalization of hypergammaglobulinemia, compared to only $19.7 \%$ in the placebo group ( $p<0.001$ ) [28]. Consistent with the effects of belimumab on immunoglobulin titers were the reductions in antibody levels, exerted in a dosedependent manner. Positive anti-dsDNA antibodies at baseline converted to negative by week 52 and normalization of anti-dsDNA by week 8 , irrespective of therapy dose, was predictive for a reduced risk of severe flares over 52 weeks. Likewise, belimumab-treated patients experienced greater positive-tonegative conversion rates of anti-Smith and antiribobosomal $\mathrm{P}$ autoantibodies as evaluated by pooled analysis. Conversely, no significant differences were recorded in antinuclear antibody titers [28].

In both BLISS trials complement levels were restored in a dose-dependent manner in a significantly greater proportion of patients treated with belimumab compared to placebo. Normalization of C3 occurred in $34 \%$ and $43.5 \%$ patients treated with $10 \mathrm{mg} / \mathrm{kg}$ belimumab compared to $14 \%$ and $20.8 \%$ of patients treated with placebo ( $\mathrm{p}=0.0005$ and $\mathrm{p}<0.01$ ) in BLISS-52 and BLISS-76, respectively [22,23,29]. Of note, there were no statistically significant effects in any of the two studies for the $1 \mathrm{mg} / \mathrm{kg}$ belimumab arm. Regarding C4 hypocomplementemia, normalization was significantly more common in both belimumab treatment arms compared to placebo and response was consistent in both trials: C4 normalization occurred in $43 \%$ and $46.4 \%$ of patients treated with $10 \mathrm{mg} / \mathrm{kg}$ belimumab compared to $19 \%$ and $17.2 \%$ of patients on SOC alone in BLISS-52 and BLISS 76, respectively. Moreover, the results were maintained through week 76 in the BLISS-76 trial and combined analysis of the two studies confirmed the individual study findings [28]. The BLISS-76 trial included a vaccine substudy to assess the effects of belimumab on vaccine-associated antibody levels in patients receiving pneumococcal or tetanus vaccine within the past 5 years. Percentages in patients maintaining antipneumococcal or antitetanus toxin IgG did not significantly differ between placebo and belimumab groups, suggesting that treatment with belimumab should not compromise immune response to vaccination [28].

\section{Organ outcomes}

Neither BLISS-52 nor BLISS-76 were designed to assess individual organ domains. Moreover, the SRI has not been evaluated in the assessment of individual organ domains in SLE; importantly it includes SELENA-SLEDAI, which does not assess activity/improvement in all organ domains. Notwithstanding these limitations, an analysis of organ outcomes is of interest. The most frequently involved organ systems were musculoskeletal (60\%), mucocutaneous (59\%), hematologic (16\%), renal (11\%) and vasculitis (9\%) [30]. No relevant discrepancies between the placebo and belimumab groups were noted at baseline. Pooled analysis $(n=1684)$ showed that belimumab versus standard of care alone was associated with significant improvement especially in the mucocutaneous and musculoskeletal organ domains. Likewise, patients treated with belimumab experienced numerically less disease worsening across several organ domains compared to placebo [31].

\section{Renal disease}

Although severe nephritis represented an exclusion criterion, $10-20 \%$ patients with renal involvement were included in both trials [22,23]. Of these, around half had significant lupus nephritis (proteinuria $\geq$ $2 \mathrm{~g}$ per day). Pooled analysis of both trials revealed a trend towards improvement at 52 weeks. Numerically lower renal flare rates $(1.4 \%$ in the belimumab $10 \mathrm{mg} / \mathrm{kg}$ arm, $2.5 \%$ in the belimumab $1 \mathrm{mg} / \mathrm{kg}$ arm, and $2.8 \%$ in the placebo arm), higher renal remission rates, and greater reductions in proteinuria levels (median \% reduction in proteinuria: $27.5 \%$ in the placebo arm, $48.3 \%$ in the $1 \mathrm{mg} / \mathrm{kg}$ belimumab arm, and $39.1 \%$ in the $10 \mathrm{mg} / \mathrm{kg}$ belimumab arm) were reported in the belimumab treatment arms compared to placebo. The type (i.e., proteinuric versus nephritic) or severity of renal flares was not reported. Patients more likely to respond receiving standard of care therapy were those with highly serologically active disease (low $\mathrm{C} / \mathrm{anti}-\mathrm{dsDNA}+$ ) at baseline. The relative rate of any improvement with belimumab $10 \mathrm{mg} / \mathrm{kg}$ versus placebo was $12.8 \%$ (OR 1.69) in the low complement/anti-dsDNA+ 


\section{Candidate patients for belimumab}

Residual disease activity

\section{Refractory disease}

High risk for flares
Patients with moderate to high disease activity (i.e. SLEDAI $\geq 6$ in spite of maximum standard of care immunosuppressive therapy. Higher disease activity (i.e. SLEDAI $\geq 8$ ) is associated with more robust responses especially if associated with serologic activity (anti-dsDNA positivity, low complement) Patients unable to taper steroids to acceptable doses for chronic use

Patients who have failed to respond or are intolerant to available standard of care therapies Patients with high serological activity /residual disease despite maximum standard of care. Patients with a history of recurrent flares

High risk for disease Long-standing disease with multiple organ involvement progression

Predictors of response Higher disease activity SELENA- SLEDAI $\geq 10$ and corticosteroid treatment to belimumab

subgroup compared to 7\% (OR 1.33) in the overall population [32]. These data, albeit interesting, need to be interpreted with caution. The studies were not designed to assess renal outcome. Renal outcomes are difficult to assess in a population who receives standard of care with established efficacy on renal involvement (like AZA or MMF - which were allowed in the trial). However, any potential improvement in renal outcomes in this context represents an encouraging result. Further studies with effect on kidney disease as an individual endpoint are warranted in order to define renal response to belimumab.

\section{Who should we treat with belimumab?}

Available data from the BLISS trials allow us to draw preliminary conclusions regarding the average SLE patient that may be a candidate for therapy with belimumab. To this end, suitable patients should be considered to be those who are seropositive and with at least moderate disease severity (SLEDAI > 6) despite receiving optimal treatment [33]. Such patients with smoldering disease are not uncommon in clinical practice and, more importantly, they tend to accumulate damage over time. Belimumab may be used as add-on therapy in patients with residual activity or who receive unacceptably high doses of steroids (e.g. unable to taper below $10 \mathrm{mg} /$ day). Additionally, its positive trend toward reduction of severe flares justifies its use for prevention in patients with frequent relapses. The fact that a quarter of patients were at flare at the initiation of the drug also suggests that the drug could be potentially useful for the management of moderate flares. More evidence is clearly needed regarding efficacy in different organ systems and of course in the setting of lupus nephritis. The positive trend for belimumab in patients with renal involvement justifies subsequent testing in a population of patients with this manifestation. Until then however, belimumab should not be used to treat SLE with severe renal or central nervous system involvement. In both BLISS-52 and -76 Black patients were underrepresented. In spite of this, the lower efficacy of BLyS blockade in this ethnic group is discouraging; potential use of the drug in African Americans will require better documentation of potency.

\section{Developing a new drug for lupus: challenges and pitfalls}

The lupus medical community has struggled for years to develop disease activity and treatment response measures that can both encompass the wide spectrum of clinical manifestations while, at the 
same time, reflect disease severity. The most widely used instruments in clinical trials are the SELENASLEDAI (SS) and the BILAG. Yet, these measures remain unsatisfactory. The SS score for instance, is used to evaluate improvement in disease activity, but it measures only the complete resolution of the assessed parameter and not partial responses. Thus, in the setting of a trial, clinically significant partial responses could be underestimated. Belimumab was both the engine and the beneficiary of the development of a more complex instrument of measure designed to be used as primary efficacy endpoint in lupus trials, the SRI. Utilizing the SRI, both BLISS-52 and BLISS-76 met their primary endpoints; clinical relevance was reached and pooled analysis of the two trials yielded greater differences at week 52 for both belimumab treatment arms.

\section{Background therapy}

Regulating background therapy in a clinical trial is crucial for the success or failure of a new biologic. To prove efficacy, in the ideal stage for a new drug, concomitant medication would only have a backup rescue role, but this is not acceptable at present. Concurrent steroids and immunosuppressants have been permitted in all trials in unstandardized protocols, as is considered standard of care in local medical practice. The comparison arm in lupus trials includes heterogeneous drugs (hydroxychloroquine, azathioprine, methotrexate, mycophenolate mofetil), administered in various doses, in mono- or combination therapy, with increases of steroids or immunosuppressants allowed for certain periods of time at study entry. In this complex setting, the bar was raised very high for a novel therapy: to prove efficacy on top of a maximal standard of care with so far unapproved drugs. Moreover, the effect of an additional drug may be additive, synergic, redundant or antagonistic; the exact influence of an immunosuppressant on a biological has not been determined.

In the phase II of trial of belimumab a 60-day stable standard of care was requested, which was afterwards allowed to be adapted upon clinical need. This left no room for belimumab to prove its efficacy. During BLISS-52 and BLISS-76, the period of stable therapy preceding the first infusion of belimumab was narrowed to 30 days, while increases in concomitant medication were limited to the first 16 weeks of trial for antimalarials and immune suppressants [22,23]. However, the addition of a new immunosuppressant was also permitted in the first 16 weeks, an intervention that might have accounted for the high response rates of the placebo group.

\section{Target population}

The success of a clinical trial relies upon the appropriate selection of the target population. From a clinical standpoint, lupus patients at most need of a biologic therapy are those who cannot be successfully managed by standard of care alone. These are severe or refractory lupus cases, patients requiring unacceptable doses of steroids or in whom certain immunosuppressants are contraindicated. One of the main limitations of the belimumab clinical trials was that they excluded the patients "in need" and addressed only mild-to-moderate cases (as well as mucocutaneous and musculoskeletal organ involvement versus renal or central nervous system involvement). As a consequence, clinical improvement could not be detected in patients with low disease activity, which was managed by the increase of steroids or changes in immunosuppressants during the first months of the trial. Likewise, secondary endpoints were flare rate and time to flare, yet both BLISS trials, especially BLISS-76, included patients with a reduced probability to flare. As a result, to show a statistically significant reduction in severe flare rates with belimumab, the size of the study had to be extended. Pooled analysis of both BLISS trials suggested that belimumab may be particularly effective in patients with higher disease activity at baseline (characterized by a SELENASLEDAI $\geq 10$, anti-dsDNA positivity or hypocomplementemia), or in patients requiring treatment with corticosteroids [33]. These findings may further help define the profile of patients with enhanced potential to response to a given biological therapy in clinical practice or future trials. 


\section{Cost-effectiveness of belimumab}

Although approved by the American Food and Drug Administration (FDA), belimumab was recently rejected by the British National Institute for Health and Clinical Excellencence (NICE) and the German Institute for Quality and Efficiency in Health Care (IQWiG). Its licensed indication was as addon therapy in adult patients with active autoantibody SLE who have a high degree of disease activity despite standard of care. NICE reported that according to the evidence of the BLISS trials, belimumab did not provide enough health benefit for patients in view of the cost of the add-on therapy compared to standard care alone. The manufacturer's cost-effectiveness analysis estimated an incremental costeffectiveness ratio (ICER, the cost of the drug in relation to how well it works) without the patient access scheme of 2-3 times the normally accepted threshold range in Great Britain. However, subsequent analysis taking into account the steroid sparing effect, a SELENA-SLEDAI score of greater than or equal to 6 (instead of 4 ) and the health effects discount rate lowered from 3.5\% to 1.5\%, considerably lowered the costs by half. The use of belimumab in clinician judgment-based situations was not discussed.

\section{Conclusions}

Lupus has long proved difficult to tackle. After years of disappointing trials and results, at long last the disease shows its first signs of yielding to biological therapy. Despite the moderate benefit of belimumab, the methodology of the BLISS trials has provided new insights in designing trials for SLE and has paved the way for greater breakthroughs in the near future.

\section{Aknowledments}

We thank Dr. George Bertsias for critical review of the manuscript.

Questions for future researches

In our opinion important questions for the future include, but are not limited to the following:

- Does the rather modest treatment effect of belimumab reflect only the population studied and/ or background therapy or the drug has a modest effect thus qualifying best as maintenance of remission/prevention of flare agent?

- Lupus patients accumulate significant damage as a result of steroid use. While studies thus far have shown a significant steroid-sparing effect, the impact of this in damage accrual needs to be better documented. Could belimumab be used in a context of a steroid-free lupus regimen?

- What is the impact of belimumab in the treatment of lupus with moderate or severe major organ involvement (nephritis, thrombocytopenia, neuropsychiatric disease) or in patients with antiphospholipid syndrome?

- How could belimumab's cost-effectiveness be improved? And how cost-effective would belimumab prove to be, if the analysis was realigned with how clinicians would prescribe belimumab in clinical setting (e.g. patients with a SELENA-SLEDAI greater than 10, possible discontinuation) and adjusted to the steroid sparing effect? Lupus is associated with substantial annual direct cost burden at par with other major rheumatologic diseases such as rheumatoid arthritis. There is a substantial economic burden, in terms of health care resource utilization as well as losses of productivity due to work capacity impairment. Little research has been done examining costs associated with specific treatments or cost variation by disease severity and disease manifestations. Future research elucidating the causes in variation of costs will help in the appraisal of emerging therapies and in developing clinical management strategies. 


\section{The review in brief}

Clinical question Systemic lupus erythematosus is the prototypic autoimmune disease characterized by involvement of several organs of variable severity. While the care of lupus has improved, existing therapies have significant side-effects while a considerable number of patients do not respond or flare after responding. This review aims at describing the role and characteristics of belimumab, a novel biologic drug targets BlyS

Type of review Narrative

Search of the Medline search for English-language articles using the following keywords: belimumab, systemic lupus literature erythematosus, and randomized controlled trials

Conclusions Belimumab is a molecule with a major role in the pathogenesis of lupus decreasing disease activity and preventing flares-especially major flares of the disease

Limitations The review covers safety issues to a lesser extent

\section{References}

1. Huscher D, Merkesdal S, Thiele K, et al. Cost of illness in rheumatoid arthritis, ankylosing spondylitis, psoriatic arthritis and systemic lupus erythematosus in Germany. Ann Rheum Dis 2006; 65: 1175-83; http://dx.doi.org/10.1136/ard.2005.046367

2. Slawsky KA, Fernandes AW, Fusfeld L, et al. A structured literature review of the direct costs of adult systemic lupus erythematosus in the US. Arthritis Care Res (Hoboken) 2011; 63: 1224-32; http://dx.doi.org/10.1002/acr.20502

3. Merrill JT, Neuwelt CM, Wallace DJ, et al. Efficacy and safety of rituximab in moderately-to-severely active systemic lupus erythematosus: the randomized, double-blind, phase II/III systemic lupus erythematosus evaluation of rituximab trial. Arthritis Rheum 2010; 62: 222-33; http://dx.doi. org/10.1002/art.27233

4. Rovin BH, Furie R, Latinis K, et al. Efficacy and safety of rituximab in patients with active proliferative lupus nephritis: the Lupus Nephritis Assessment with Rituximab study. Arthritis Rheum 2012; 64: 1215-26; http://dx.doi.org/10.1002/art.34359

5. Ginzler EM, Wax S, Rajeswaran A, et al. Atacicept in combination with MMF and corticosteroids in lupus nephritis: results of a prematurely terminated trial. Arthritis Res Ther 2012; 14: R33; http:// dx.doi.org/10.1186/ar3738

6. Mackay F, Schneider P. Cracking the BAFF code. Nat Rev Immunol 2009; 9: 491-502; http://dx.doi. org/10.1038/nri2572

7. Cancro MP, D'Cruz DP, Khamashta MA. The role of B lymphocyte stimulator (BLyS) in systemic lupus erythematosus. J Clin Invest 2009; 119: 1066-73; http://dx.doi.org/10.1172/JCI38010

8. von Bulow GU, van Deursen JM, Bram RJ. Regulation of the T-independent humoral response by TACI. Immunity 2001; 14: 573-82; http://dx.doi.org/10.1016/S1074-7613(01)00130-3

9. Castigli E, Scott S, Dedeoglu F, et al. Impaired IgA class switching in APRIL-deficient mice. Proc Natl Acad Sci U S A 2004; 101: 3903-8; http://dx.doi.org/10.1073/pnas.0307348101

10. Hostmann A, Jacobi AM, Mei H, et al. Peripheral B cell abnormalities and disease activity in systemic lupus erythematosus. Lupus 2008; 17: 1064-9

11. Khare SD, Sarosi I, Xia XZ, et al. Severe B cell hyperplasia and autoimmune disease in TALL-1 transgenic mice. Proc Natl Acad Sci U S A 2000; 97: 3370-5; http://dx.doi.org/10.1073/pnas.050580697

12. Ramanujam M, Wang X, Huang W, et al. Similarities and differences between selective and nonselective BAFF blockade in murine SLE. J Clin Invest 2006; 116: 724-34; http://dx.doi.org/10.1172/ JCI26385 
13. Ramanujam M, Bethunaickan R, Huang W, et al. Selective blockade of BAFF for the prevention and treatment of systemic lupus erythematosus nephritis in NZM2410 mice. Arthritis Rheum 2010; 62: 1457-68; http://dx.doi.org/10.1002/art.27368

14. Baker KP, Edwards BM, Main SH, et al. Generation and characterization of LymphoStat-B, a human monoclonal antibody that antagonizes the bioactivities of B lymphocyte stimulator. Arthritis Rheum 2003; 48: 3253-65; http://dx.doi.org/10.1002/art.11299

15. Cheema GS, Roschke V, Hilbert DM, et al. Elevated serum B lymphocyte stimulator levels in patients with systemic immune-based rheumatic diseases. Arthritis Rheum 2001; 44: 1313-9; http:// dx.doi.org/10.1002/1529-0131(200106)44:6<1313::AID-ART223>3.0.CO;2-S

16. Petri M, Stohl W, Chatham W, et al. Association of plasma B lymphocyte stimulator levels and disease activity in systemic lupus erythematosus. Arthritis Rheum 2008; 58: 2453-9; http://dx.doi. org/10.1002/art.23678

17. Collins CE, Gavin AL, Migone TS, et al. B lymphocyte stimulator (BlyS) isoforms in systemic lupus erythematosus: disease activity correlates better with blood leukocyte BLyS mRNA levels than with plasma BLyS protein levels. Arthritis Res Ther 2006; 8: R6

18. Furie R, Stohl W, Ginzler EM, et al. Biological activity and safety of belimumab, a neutralizing anti-B-lymphocyte stimulator (BLyS) monoclonal antibody: a phase I trial in patients with systemic lupus erythematosus. Arthritis Res Ther 2008; 10: R109; http://dx.doi.org/10.1186/ar2506

19. Scott LJ, Burness CB, McCormack PL. Belimumab: a guide to its use in systemic lupus erythematosus. BioDrugs 2012; 26: 195-9; http://dx.doi.org/10.2165/11209060-000000000-00000

20. Wallace DJ, Stohl W, Furie RA, et al. A phase II, randomized, double-blind, placebo-controlled, dose-ranging study of belimumab in patients with active systemic lupus erythematosus. Arthritis Rheum 2009; 61: 1168-78; http://dx.doi.org/10.1002/art.24699

21. Furie RA, Petri MA, Wallace DJ, et al. Novel evidence-based systemic lupus erythematosus responder index. Arthritis Rheum 2009; 61: 1143-51; http://dx.doi.org/10.1002/art.24698

22. Navarra SV, Guzmán RM, Gallacher AE, et al. Efficacy and safety of belimumab in patients with active systemic lupus erythematosus: a randomized, placebo-controlled, phase 3 trial. Lancet 2011; 377: $721-31$

23. Furie R, Petri M, Zamani O, et al. A phase III, randomized, placebo-controlled study of belimum$\mathrm{ab}$, a monoclonal antibody that inhibits B lymphocyte stimulator, in patients with systemic lupus erythematosus. Arthritis Rheum 2011; 63: 3918-30; http://dx.doi.org/10.1002/art.30613

24. Petri M, Levy RA, Merill JT, et al. Belimumab, a BLyS-specific inhibitor, reduced disease activity, flares, and prednisone use in patients with seropositive SLE: combined efficacy results from the phase 3 BLISS-52 and -76 studies. Arthritis Rheum 2010; 62 Suppl 10: 190

25. Cervera R, Furie R, Levy R, et al. Relationship of belimumab treatment response with corticoid use and severe flares in patients with SLE: BLISS-52 and BLISS-76. Ann Rheum Dis 2011; 70 (Suppl 3): 321

26. Navarra S, Ilianova E, BaeSC, Guzman R, Tanasescu C, et al. Belimumab, a BLyS-specific inhibitor, reduced disease activity, flares, and steroid use in patients with seropositive systemic lupus erythematosus (SLE): BLISS-52 study. Ann Rheum Dis 2010; 69 (Suppl 3): 556

27. van Vollenhoven RF, Zamani O, Wallace DJ, et al. Belimumab, a BLyS-specific inhibitor, reduces disease activity and severe flares in seropositive SLE patients: BLISS-76 study. Ann Rheum Dis 2010; 69 (Suppl 3): 74

28. Stohl W, Hiepe F, Latinis KM, et al. Belimumab reduces autoantibodies, normalizes low complement, and reduces select B-cell populations in patients with systemic lupus erythematosus. Arthritis Rheum 2012; Jan 24; doi: 10.1002/art.34400. [Epub ahead of print]

29. Levy RA, Ilianova E, Ionescu R, et al. Belimumab, a BLyS-specific inhibitor, significantly reduced autoantibodies and normalized low complement in patients with seropositive systemic lupus erythematosus (SLE): BLISS-52 study. Ann Rheum Dis 2010; 69 (Suppl 3): 556 
30. Manzi S, Sánchez-Guerrero J, Merrill JT, et al. Belimumab, a BLys-specific inhibitor, reduces disease activity acoss multiple organ domains: combined efficacy results from the phase 3 BLISS-52 and -76 studies. Arthritis Rheum 2010; 62 (Suppl 10): 607

31. D’Cruz D, Manzi S, Sánchez-Guerrero J, et al. Belimumab reduced disease activity across multiple organ domains in patients with SLE: combined results from BLISS-52 and BLISS-76. Ann Rheum Dis 2011; 70 Suppl 3: 318

32. Dooley MA, Houssiau F, Aranow C, et al. Effect of belimumab treatment on renal outcomes: results from phase 3 belimumab clinical trials in patients with systemic lupus erythematosus. Arthritis Rheum 2011; 63 (Suppl 10): 2472

33. van Vollenhoven RF, Petri MA, Cervera R, et al. Belimumab in the treatment of systemic lupus erythematosus: high disease activity predictors of response. Ann Rheum Dis 2012 Mar 7 [Epub ahead of print] 